Из доказанного следует, что только у вырождающихся поверхностей могут быть особые точки. Итак, только в случае вырождающейся поверхности второго порядка и только в ее особой точке $M_{0}=\left(x_{0}, y_{0}, z_{0}\right)$ касательная плоскость к поверхности (1) оказывается неопределенной. Попутно мы доказали, что при выполнении условий (10) условия $\left(1_{0}\right)$ и (12) эквивалентны между собой.

$$
* * *
$$

1. Энциклопедия электронной математики. Том1, арифметика, М.2007

2. Александров П.С. Курс аналитической геометрии и линейной алгебры. 1979

3. Энциклопедия электронной математики. Том 1, арифметика, М.2007 г.

4. Бюшгенс С.С. Дифференциальная геометрия - М.:Наука,2008 г.

5. Погорелов А.В. Дифференциальная геометрия - М.:Наука,2009 г.

\title{
Гацаева P.C-A. \\ Методы псевдовогнутого анализа в теории нелинейных уравнений и неравенств Вольтера
}

ФГБОУ ВО «Чеченский государственный университет имени А.А. Кадырова» (Россия, Грозный)

doi: 10.18411/trnio-01-2022-57

\section{Аннотация}

В статье реализуется применение, так называемого, метода псевдовогнутого анализа к многомерным нелинейным интегральным уравнениям Вольтера с нелинейностью псевдовогнутого вида.

Ключевые слова: псевдовогнутый, многомерный, нелинейный, интегральный, уравнение, аргумент.

\section{Abstract}

The article implements the application of the so-called method of pseudo-concave analysis to multidimensional nonlinear Voltaire integral equations with nonlinearity of pseudo-concave form.

Keywords: pseudo-concave, multidimensional, nonlinear, integral, equation, argument.

В работе реализуется применение, так называемого, метода псевдовогнутого анализа к многомерным нелинейным интегральным уравнениям Вольтера с нелинейностью псевдовогнутого вида.

Рассмотрим многомерное нелинейное интегральное уравнение Вольтера

$$
u(x)=\int_{0}^{x} k[x, t, u(t)] d t+f(x)
$$

Всюду в дальнейшем полагаем:

$$
\begin{gathered}
x=\left(x_{1}, \ldots, x_{n}\right), t=\left(t_{1}, \ldots, t_{n}\right), \in(0,1), l=\left(l_{1}, \ldots, l_{n}\right), l_{1}>0 \\
u(x)=\left\{\left(u_{1}(x), \ldots, u_{n}(x)\right\} \in K_{+},\right. \\
f(x)=\left\{\left(f_{1}(x), \ldots, f_{n}(x)\right\} \in K_{+},\right.
\end{gathered}
$$

где $K_{+}$-конус положительных при $x>0$ функций ,частично упорядоченного этим конусом векторного В-пространства $X=C$ или $L_{P}, 1 \leq p \leq \infty$,

$$
K_{+}=\{u(x): u(x) \in X, u(x)>0, x>0\}
$$

Запись $u, f \in(0, L], L=\left(L_{1}, \ldots, L_{n}\right), L_{1}>0$, означает, что значения $и$ или $f$ при фиксированных аргументах принадлежат параллелепипеду $(0, L]$ евклидова пространства $E^{n}$.Все операции и соотношения между векторами или матрицами понимаются покомпонентно. Например, $u^{\gamma}=\left\{u_{1}^{\gamma}, \ldots, u_{n}^{\gamma}\right\}, \gamma(u)=\left\{\left(\gamma\left(u_{1}\right), \ldots, \gamma\left(u_{n}\right)\right\}\right.$ и т.д. 
Изучению уравнений Вольтера вида (1) посвящена обширная литература (см.библиографию [7]), при этом функции $f$ и $k$ предполагаются в основном непрерывными в своих областях определения и как правило, предполагается ,что функция $k(x, t, u)$ неотрицательна монотонно возрастает по аргументу $u$.

Непрерывность гарантирует локальное существование решений, а монотонность даёт возможность находить априорные оценки решений и задать конусной отрезок $\left\langle v_{0}, w_{0}\right\rangle$, в котором расположены все нужные решения уравнения (1).

В настоящей работе реализован подход, при котором при более слабых условиях чем непрерывность, монотонность ядра $k$ по аргументу $u$ не предполагается, а вводится более общее условие принадлежности функции $k(x, t, u)$ по аргументу $u$, так называемому, классу псевдовогнутой нелинейности типа (А) или (П).

\section{1. Метод псевдовогнутой нелинейности.}

Пусть $\gamma(u)$ и $\lambda(u)$-непрерывные, монотонные положительные на $(0, \mathrm{~L}], \quad \mathrm{L}<$ $\infty(0, \mathrm{~L}), \mathrm{L} \leq \infty$ заданные функции .

Классы типа (A).

ЧерезА $\mathrm{A}^{\gamma(u)}, \mathrm{A}_{\gamma(u)}$ обозначим классы непрерывных и положительных на $(0, \mathrm{~L}]$ функций $\varphi(u)$ таких ,что $\frac{\varphi(u)}{\gamma(u)}$ почти убывает на $(0, \mathrm{~L}]$.

Классы типа (П).

$\Pi^{\gamma(u)} \subset \mathrm{A}^{\gamma(u} \Pi_{\lambda(u)} \subset \mathrm{A}_{\lambda(u)}$ подклассы функции $\varphi(u)$, для каждой из которых существует функции $\mu_{\varphi}(u), v_{\varphi}(u)$ типа модулей непрерывности такие, что соответственно $\frac{\varphi(u)}{v(u) \mu_{\varphi}(u)}$ так же почти возрастает, также почти убывает. обозначают и так далее классы типа обладают замечательных свойств: свойства первое. $\frac{\varphi(u)}{\lambda(u)} v_{\varphi}(u)$ так же почти убывает. $\Pi_{\lambda(u)}^{\gamma(u)}=\Pi^{\gamma(u)} \cap \Pi_{\lambda(u)}$.

При $\gamma(u)=u^{\gamma}, \lambda(u)=u^{\lambda}, \gamma, \lambda \in R$ обозначают $\mathrm{A}^{\gamma}, \mathrm{A}_{\lambda}, \mathrm{A}_{\lambda}^{\gamma}, \Pi_{\lambda}^{\gamma}$ и т, д.

Классы типа (А) и (П) обладают рядом замечательных свойств:

Свойство 1.Непосредственно из определения следует, что существуют $d<0$ и $C>$ 0 такие, что справедливы неравенств

$$
\begin{gathered}
d \lambda(u) \leq \varphi(u) \leq C \gamma(u) \text { для } \varphi \in \mathrm{A}_{\lambda(u)}^{\gamma(u)} \\
d \lambda(u) \leq \varphi(u) \leq C \gamma \lambda(u) \quad \varphi \in \Pi_{\lambda(u)}^{\gamma(u)} \\
\text { или } \\
d \lambda(u) / v_{\varphi}(u) \leq \varphi(u) \leq C \gamma(u) \mu_{\varphi}(u) .
\end{gathered}
$$

Неравенства (2) и(3) позволяют эффективно применять классы типа (А)и (П) в теории нелинейных интегральных неравенств уравнений .

Свойства 2. Для любой функции $\varphi(u) \in \mathrm{A}_{\lambda}^{\gamma} \Pi_{\lambda}^{\gamma}$ существует функция $\varphi^{*}(u)$ типа модулей непрерывности, эквивалентная $\varphi$ такая, что $\frac{\varphi^{*}(u)}{u^{\gamma}}$ возрастает, $\frac{\varphi^{*}(u)}{u^{\lambda}}$ убывает и $\omega_{\lambda}\left(\varphi^{*}, \delta\right) \sim \varphi^{*}(\delta)$. Подклассы таких функций будем обозначать $(\mathrm{A} *),(\Pi *)$.

Лемма 1. Пусть измеримая функция $k(x, t, u)$ неотрицательна на $(0,1] \times(0,1]$,

кроме того по аргументу и принадлежит классу $\mathrm{A}_{\lambda(u)}^{\gamma(u)}$ или $\Pi_{\lambda(u)}^{\gamma(u)}$.Тогда существует функции

$$
\begin{gathered}
P(x, t) \cdot \lambda(u) \leq k(x, t, u) \leq \mathrm{Q}(x, t) \gamma(u) \text { для }(\mathrm{A}) \\
P(x, t) \cdot \lambda(u)<k(x, t, u)<\mathrm{Q}(x, t) \gamma(u)
\end{gathered}
$$

или $P(x, t) \cdot \lambda(u) / v(u) \leq k(x, t, u) Q(x, t) \gamma(u) / \mu(u)$ для П

Доказательство. Доказательства неравенства (4) и (5) получаем непосредственно из определения классов типа (А )и типа (П ) соответственно с учетом неравенств (2) и (3) . 
Свойства функций $\mathrm{P}(\mathrm{x}, \mathrm{t})$ и $\mathrm{Q}(\mathrm{x}, \mathrm{t})$ полностью определяются свойствами функций $k(x, t, u$ ии $\gamma(u), \lambda(u)$.

Запишем следующие уравнения:

$$
\begin{aligned}
& V(x)=f(x)+\int_{0}^{x} P(x, t) \lambda[v(x)] d t, x>0, x \in R^{n}, v=A_{1} u+f \\
& \omega(x)=f(x)+\int_{0}^{x} \mathrm{Q}(x, t) \lambda[\omega(x)] d t, x>0, x \in R^{n}, v=A_{2} u+f
\end{aligned}
$$

где $P(x, t)$ и Q $(x, t)$ функции из неравенств(2) и (3), $\gamma(u) \quad u \quad \lambda(u)$ монотонны.

Теорема 2. Пусть $v_{0}(x)$ и $\omega_{0}(x)$ нижнее и верхнее решения уравнений (6) и (7) соответственно; функция

$f(x) \in K_{+} \subset X(0 \leq t \leq L<\infty,|u|<\delta,|\delta| \leq \infty ; \quad$ вектор-функция $\quad k \quad(x, \quad t, u \quad)$ удовлетворяет условиям Каратеодори, и по аргументу и принадлежит классу для $\mathrm{A}_{\lambda(u)}^{\gamma(u)}$ при всех $x$ почти при всех t. Тогда все решения исходного уравнения (1) один принадлежат конусному отрезку $<v_{0} \omega_{0}>$

\section{Доказательство.}

На основании леммы 1, используя неравенства (2), из исходного уравнения (1) получаем нестрогие интегральные неравенства.

$$
\begin{aligned}
& u(x) \geq f(x)+\int_{0}^{x} P(x, t) \lambda[u(x)] d t, \\
& u(x) \leq f(x)+Q \int_{0}^{x}(x, t) \gamma[u(x)] d t
\end{aligned}
$$

На основании теоремы об интегральных неравенствах из неравенств (8) и (9) получаем, что нижнее решение $v_{0}(x)$ уравнения (6) с монотонным оператором $A_{1}$, и верхнее решение $\omega_{0}(x)$ уравнения (7) с монотонным оператором $A_{2}$, являются априорными оценками искомых решений $u(x)$ уравнения (1), удовлетворяют неравенствам $v_{0}(x) \leq u(x) \leq \omega_{0}(x)$

и задают конусный отрезок $<v_{0}, \omega_{0}>$ в котором исследуются решения исходного уравнения известными классическими методами.

Замечание 1. Если в теореме 2 вместо класса $\mathrm{A}_{\lambda(u)}^{\gamma(u)}$ взять класс $\Pi_{\lambda(u)}^{\gamma(u)}$, то в конусном отрезке $<v_{0}, \omega_{0}>$ функция $v_{0}(x)$-любое решение уравнения $(6), \omega_{0}(x)$-любое решение уравнения (7).

Нахождение конусного отрезка $<v_{0}, \omega_{0}>$ - один из важных этапов исследования нелинейных интегральных уравнений Вольтера. Чтобы получить оценки решений, тем более явные оценки решений приходится решать интегральные уравнения Вольтера, что не всегда удаётся, даже случае, когда они линейны. Поэтому для нас является важным то, что этот метод дает возможность определить эти оценки через решения более простых уравнений и неравенств, к которым удаётся свести их в случае псевдовогнутой нелинейности типа (А) или (П).

\section{***}

1. Барин Н.К., Стечкин С.Б. Наилучшие приближения и дифференциальные свойства двух сопряженных функций.Тр. Матем. общ-ва., 1956.Т.5.С. 483- 522.

2. Целюк 3.Б.Нелинейные уравнения Вольтера с неубывающим ядром. Изв.вузов.Математика 1995 8(399).C.74- 77.

3. О некоторых многомерных интегральных неравенствах. Дифференц. уравнения 1983. Т.19. 10 С. 1828 -1830.

4. АзбелевН.В., ЦалюкЗ.Б.Об интегральных неравенствах. 1.Матем.сб.1962. Т. 56. 3 .С.325-342

\section{Гацаева P.C-A.}

\section{Методы решения алгебраических, нелинейных и трансцендентных уравнений}

ФГБОУ ВО «Чеченский государственный университет имени А.А. Кадырова» doi: 10.18411/trnio-01-2022-58 